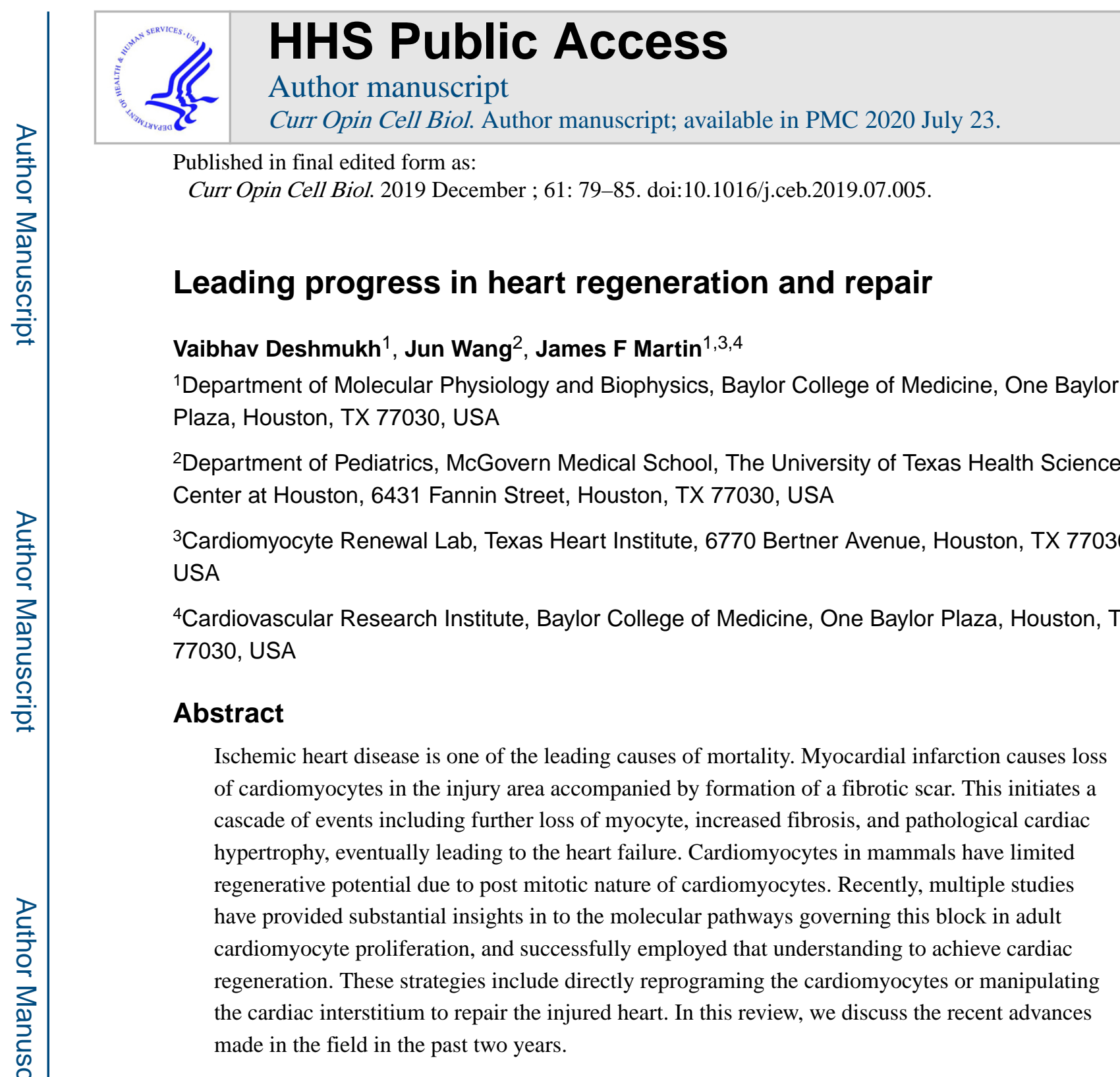

\title{
Introduction
}

Heart failure (HF) is a leading cause of mortality worldwide. Although HF can be treated with drugs to control symptoms [1] or with ventricular assist device implantation, the only definitive treatment is heart transplantation, which is limited by the scarcity of donor hearts [2]. Therefore, new approaches to treat HF are necessary.

The human heart is one of the least regenerative organs, with an adult cardiomyocyte (CM) renewal rate of less than $1 \%$ [3]. After the massive loss of CMs caused by an injury such as myocardial infarction (MI), the heart is not capable of self-repair. In contrast to the adult human heart, the hearts of adult zebrafish, lower vertebrates, neonatal mice, and neonatal swine can regenerate after injury [4-6], showing regenerative responses such as the cell-

Corresponding author: Martin, James F (jfmartin@bcm.edu).

Conflict of interest statement

None declared. 
cycle re-entry of existing myocytes and the repopulation of the scar area [7]. To develop new therapeutic options for patients with HF, we must understand the genetic mechanisms of heart regeneration so that they can be manipulated to aid in human heart repair. In this review, we discuss recent studies that have advanced our understanding of heart regeneration and repair (Figures 1 and 2).

\section{Cardiomyocyte proliferation and heart regeneration}

The Hippo pathway is an evolutionarily conserved kinase cascade that regulates organ size and has been shown to have important functions in cardiac development, homeostasis, pathology, and regeneration [8,9]. In mice, the conditional CM-specific deletion of Salv1-a core Hippo signaling component-after MI induces the cell cycle re-entry of existing CMs, leading to the reversal of heart failure. Furthermore, the delivery of short hairpin RNA (shRNA)-specific for Salv1 via adeno-associated virus 9 (AAV9) either immediately or a few weeks after cardiac injury preserves or improves heart function, respectively; promotes cell cycle re-entry in CMs; and decreases infarct size [10*0], indicating that the delivery of AAV9-Salv-shRNA may be a potential strategy for treating patients with HF. AAV9 selectively infects cardiac muscles in mice and humans and has been shown to be clinically safe. In addition, the overexpression of an active form of Hippo signaling downstream effector Yap (i.e. Yap-5SA) in CMs has provided insight into the mechanisms of Yapinduced cell cycle re-entry in postmitotic adult CMs. Yap-5SA reverses the chromatin accessibility profile in adult CMs to a fetal-like state [11]. During cardiac differentiation, chromatin rearrangement leads to the inaccessibility of loci responsible for the expression of pluripotency factors and the increased accessibility of lineage-specific genes [12], suggesting that chromatin accessibility can be targeted to stimulate cardiac regeneration [11].

The Hippo-Yap pathway is known to be modulated by mechanotransduction, cytoskeletal tension, and matrix rigidity [8]. Extracellular matrix (ECM) cues have been shown to negatively regulate Yap activity in CMs via the dystrophin and glycoprotein complex (DGC), which is a multi-subunit complex that connects the ECM to the actin cytoskeleton. Recently, Morikawa et al. [13*] showed that phosphorylated Yap interacts with dystroglycan 1 (DAG1), a core component of the DGC, resulting in the sequestration of Yap at the plasma membrane and the inhibition of CM proliferation. A study by Bassat et al. [14*0] showed that the extracellular protein Agrin interacts with the DGC, causing its disassembly and, in turn, the disruption of Yap-DGC complex formation, thereby promoting the nuclear localization of Yap and CM proliferation. Treating mice with Agrin was shown to reduce scar size and promote cardiac functional recovery after MI [14*0. FAT atypical cadherin 4 (Fat4) and angiomotin-like protein 1 (Amotl1) can also form a complex with Yap, which leads to the sequestration of Yap at the CM cell junction, independent of Hippo signaling. The genetic deletion of Fat4 promotes increased cardiomyocyte proliferation and ventricular area in a Yap-dependent manner [15]. Nuclear translocation of Yap activates genes involved in the cell cycle, the injury response, mitochondrial quality control, the stress response, and antioxidation $\left[10^{*}, 11,14^{\bullet *}, 16,17\right]$. Genetic studies by Tao et al. [16] revealed that paired-like homeodomain-2 (Pitx2) cooperates with Yap to protect the heart from injury and to enhance regeneration by activating the antioxidant gene response. In addition, Pitx2 plays an 
important role in maintaining cellular composition during heart regeneration and in regulating the transcription of mitochondrial genes [18]. In Pitx2-deficient mouse hearts, fat accumulation was observed in the myocardium $[18,11]$.

Recently, vitamin D was identified as a CM mitogen in a large-scale compound screening performed in zebrafish by using a $\mathrm{cmlc} 2$ promoter-controlled fluorescent ubiquitin-based cell cycle indicator (FUCCI) [19*]. In zebrafish, the inhibition of vitamin D signaling by globally deleting the vitamin $\mathrm{D}$ receptor ( $V D R$ ) gene or by expressing a dominant-negative form of VDR caused stunted growth. Expressing a constitutively active form of VDR in zebrafish CMs led to cardiomegaly, suggesting that vitamin D signaling controls organ size [19*0]. Furthermore, in zebrafish treated with alfacalcidol, a vitamin D analog, CM proliferation was increased through the ErbB2 signaling axis [19*0].

\section{Cell cycle, ploidy, and heart regeneration}

In neonatal mice, CMs have a short regenerative window that is lost after the first week of birth [8]. However, the signaling pathways that govern this switch remain poorly understood. One striking difference between nonregenerative and regenerative species is ploidy. Species with higher regenerative capabilities have more mononucleated and diploid cells [20*0. CMs from neonatal mice are mostly diploid, but those from adults are mostly polyploid [21]. Mouse strains with a higher percentage of diploid CMs have shown a better regenerative response [21]. The genetic deletion of TNNI3-interacting kinase (TNNI3K), one of the factors regulating ploidy in mice, increases the number of mononucleated diploid and proliferative cardiomyocytes [21]. In zebrafish, the genetic deletion of Ect2 or the overexpression of the dominant-negative form of Ect2 (dnEct2) results in increased ploidy [22]. Furthermore, the overexpression of dnEct 2 in CMs leads to an increased number of polyploid cells [22]. After injury, zebrafish hearts overexpressing dnEct2 showed reduced cardiomyocyte proliferation and increased scar formation [22].

In an oxygen-rich environment, the postnatal switch from glycolytic to oxidative phosphorylation leads to the increased production of mitochondrial reactive oxygen species (ROS). In turn, the DNA damage response is activated, which leads to cell cycle arrest in CMs [23]. In mice exposed to long-term systemic hypoxic conditions, ROS production was shown to be decreased. Furthermore, chronic hypoxia promoted a regenerative response in mouse hearts after MI [24]. Interestingly, the cell cycle exit of CMs and the loss of regenerative capabilities have been strongly correlated with the transition from ectotherms to endotherms $\left[20^{\circ}\right]$. Immediately after birth, thyroid hormone, which is a major regulator of thermogenesis, increases by more than 50 -fold [20॰]. The inhibition of thyroid hormone with NH3, a thyroid hormone-specific inhibitor, and propylthiouracil (PTU) increased the proliferation of CMs [20*]. In addition, expressing the dominant-negative form of the thyroid hormone receptor-a (DN-Thra) in CMs increased the number of diploid cells and total CMs $\left[20^{*}\right]$. This change was accompanied by the increased expression of cell cycle and G2M checkpoint genes and the decreased expression of genes related to oxidative phosphorylation, the tricarboxylic acid (TCA) cycle, mitochondria, and muscle contraction [20*0. In addition, mice expressing DN-Thra showed improved function after ischemiareperfusion injury [20*]. In an in vivo viralmediated screen, a combination of four cell cycle 
regulators were identified (i.e. CDK1/CCNB/CDK4/CCND, or $4 \mathrm{~F}$ ) that activate cardiomyocyte proliferation [ $25^{\circ}$ ]. Replacing CDK1 and CCNB with Wee1 inhibitor (i.e. MK1775) and transforming growth factor $\beta$ (TGF- $\beta$ ) inhibitor (i.e.SB431542) or $2 \mathrm{~F} 2 \mathrm{i}$ enhanced cardiomyocyte proliferation $\left[25^{\circ}\right.$ ]. Furthermore, the viral delivery of $4 \mathrm{~F}$ and $2 \mathrm{~F} 2 \mathrm{i}$ induced cardiomyocyte proliferation, reduced scar size, and improved functional recovery after a cardiac injury $\left[25^{\circ}\right]$.

\section{MicroRNAs and heart regeneration}

MicroRNAs (miRs) are small noncoding RNAs that act as posttranscriptional regulators of gene expression. miRs have been shown to play critical roles in cardiac development, disease, and regeneration [4]. Specifically, miR-199a, miR-590, miR-17-92, miR-302-367, miR-214, and miR-222 were reported to promote CM proliferation and heart regeneration, whereas miR-15 family miRs were shown to repress CM proliferation and cardiac repair [4]. In addition, miR-128 expression was shown to gradually increase as heart development progresses from a more proliferative state to a more differentiated state. The deletion of miR-128 promotes CM proliferation and cardiac regeneration, most likely because miR-128 represses the cell cycle regulators cyclin E and cyclin-dependent kinase (CDK) through the chromatin modifier SUZ12 downregulating levels of p27 (a CDK inhibitor) [26]. As mouse heart development progresses from the embryonic to adult stage [27], miR-294 expression decreases; however, the controlled, transient expression of miR-294 leads to a regenerative response in the heart after MI. miR-294 represses Wee1 and, in turn, activates CDK1 [27],

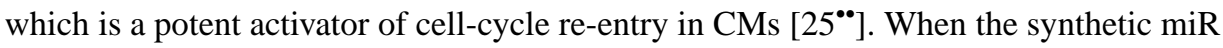
mimics miR-199a-3p and miR-590-3p were transiently delivered by using a lipid-based vehicle (i.e. RNAiMAX), significantly increased CM proliferation was observed, as well as decreased cardiac scar formation and increased cardiac function in the short term [28]. In addition, a miR-302-hydrogel complex was shown to promote CM proliferation and functional recovery after MI by directly repressing the Hippo signaling components Mst1, Lats2, and Mob1, sequentially leading to the activation of the Yap-mediated transcription

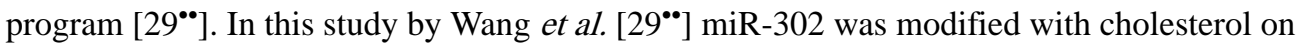
the $5^{\prime}$ end of the passenger strand to reduce electrostatic repulsion and enhance the encapsulation of miR-302 into shear thinning hydrogels before being passively internalized by cells in vitro and in vivo [4]. Liu et al. recently reported that hydrogel mediated extended delivery of extracellular vesicles (EV)secreted from the induced pluripotent stem cell derived cardiomyocytes (iPS-CM) in rats promotes cardiac recovery after an MI. These EV's where enriched in cardiac-specific miR, miR-1, and miR-133 [31“*]. The miR-17-92 cluster was also previously shown to promote CM proliferation. Recently, its individual members miR-19a/19b were found to be increased in HF patients [30], indicating their potential as therapeutic targets for HF treatment. A combination of studies in mice involving the intracardiac injection of miR-19a/19b mimics, adeno-associated virus delivery, and the systemic delivery of miR-19a/19b showed that miR-19a/19b enhance CM proliferation, reduce cardiac damage caused by MI, and improve cardiac function and repair after MI. miR-19a/19b were also shown to repress genes involved in the immune response [30]. 


\section{Immune system and heart regeneration}

The immune system plays a dual role in cardiac injury and regeneration that involves both the innate and adaptive immune systems [32]. The inflammatory response is necessary and beneficial for the initial injury response, but a prolonged inflammatory response leads to deleterious effects [32].

The adaptive immune response is composed of $\mathrm{T}$ and $\mathrm{B}$ cells, which mediate the immune response after MI [32]. The effector T cells that are activated at the proximal lymph nodes can be classified as $\mathrm{CD} 8^{+}$and $\mathrm{CD} 4^{+}$cells. $\mathrm{CD} 4^{+}$cells can be further classified as $\mathrm{TH} 1, \mathrm{TH} 2$, $\mathrm{TH} 17$, and $\mathrm{T}_{\text {reg }}$ cells, depending on the cytokines they release [32]. In zebrafish, $\mathrm{T}_{\text {reg }}$-like cells were shown to be recruited to the injury site and to promote heart regeneration. Furthermore, the conditional ablation of $\mathrm{T}_{\text {reg-like cells led to an impaired regenerative }}$ response mediated by the potent mitogen $\mathrm{Nrg} 1$ that was secreted by $\mathrm{T}_{\text {reg }}$ cells at the injury site [33]. In mammals, neonatal $\mathrm{CD}^{+} \mathrm{T}$ cells have the innate ability to become $\mathrm{T}_{\text {reg }}$ cells in response to $\mathrm{T}$ cell receptor activation for up to two weeks after birth. Recently, $\mathrm{T}_{\text {reg }}$ cells were reported to promote $\mathrm{CM}$ proliferation and to contribute to increased maternal heart size during pregnancy in mice [34*0. The depletion of $\mathrm{T}_{\text {reg }}$ cells led to increased deleterious outcomes after MI, whereas injecting $\mathrm{T}_{\text {reg }}$ cells at the site of injury elicited CM proliferation, reduced scar size, and improved heart function after $\mathrm{MI}$ in mice [34*0. $\mathrm{T}_{\text {reg }}$ cells secreted Cst7, Tnfsf11, Il33, Fgl2, Matn2, and Igf2 to promote CM proliferation. The expression of these factors in mice via AAV recapitulated CM proliferation and functional recovery [34*0]. The Hippo pathway has also been implicated in regulating $\mathrm{T}_{\text {reg }}$ cells. Mice with epicardial Yapand Taz deficiency showed a decreased number of $\mathrm{T}_{\text {reg }}$ cells in response to $\mathrm{MI}$ and exhibited increased inflammation, a larger scar area, and decreased cardiac function [35].

Macrophages play an important role in cardiac repair [32]. They are the most abundant immune cell population in the heart and compose $5 \%-10 \%$ of the non-CM population. Tissue-resident macrophages have been shown to localize in the distal atrioventricular node in mice and humans and aid in the shortening of the action potential in CMs [36]. A comparative analysis between regenerative and nonregenerative zebrafish and medaka revealed the delayed and reduced recruitment of macrophages to the infarct area in Medaka, which eventually led to impaired neutrophil clearance, scar resolution, CM proliferation, and neovascularization [37]. Delaying macrophage recruitment in zebrafish by using clodronate also led to impaired heart regeneration [37]. The heterogeneity of macrophages is one of the greatest challenges to gaining a deeper understanding of the role of macrophages in heart regeneration. In a recent study, single-cell transcriptomics studies were combined with cell fate mapping to dissect the cellular heterogeneity of cardiac macrophages during homeostasis and after injury, such as MI or cardiomyopathy [38*0. Although resident macrophages composed only $2 \%-5 \%$ of the total macrophage population in the infarct region, their depletion led to impaired cardiac wound healing and increased mortality after MI, revealing an important role for resident macrophages in cardiac repair [38*0].

The complement cascade is part of the innate immune system and has traditionally been viewed as responsible for the inflammatory response. Recently, the complement system was implicated in tissue regeneration [39]. Complement receptor C5aR1 was shown to be 
upregulated in the regenerating hearts of zebrafish, axolotl, and mice [40]. In all three species, the inhibition of C5aR1 led to decreased CM proliferation and increased scar size [40].

\section{Vasculogenesis and heart regeneration}

In the infarcted region of the heart, angiogenesis is part of the healing response and improves patient prognosis [41]. In zebrafish, cardiac regeneration is accompanied by the rapid revascularization of the infarcted region. Furthermore, ablation of this angiogenic response leads to reduced cardiomyocyte proliferation and nonresolution of the scar [42]. In Hippo-deficient CMs, which efficiently regenerate after MI, genes involved in vasculogenesis are upregulated $\left[10^{\circ}\right]$. In a recent study, whole-organ imaging was used to identify the increased formation of a collateral artery network in the watershed area of regenerative neonatal (P2) mouse hearts, which was absent in nonregenerative mouse hearts $\left[43^{\circ}\right]$. These collateral arteries are formed by the migration of arterial endothelial cells

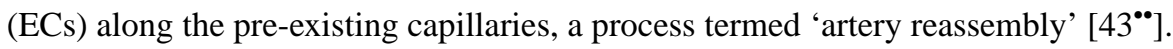
CXCL12 is a chemo- tactic ligand inducible by hypoxic conditions and is crucial for coronary EC migration during mouse development [43"*]. CXCL12 was detected in the watershed region in the hypoxic but not the normoxic neonatal mouse heart [43*0]. Furthermore, after MI, CXCL12 was detected in the watershed region proximal to the scar but not in the distal region of neonatal mouse hearts [43**]. The deletion of Cxcl12 or its receptor $\mathrm{Cxcr} 4$ impairs EC migration and collateral artery formation in the neonatal mice heart after MI [43*0]. In addition, the exogenous application of CXCL12 at P7 in mice stimulated the formation of collateral arteries, which was diminished postnatally [43*0]. Cxcl12 has been shown to be expressed primarily in the epicardium, which supports the expansion of vascular networks toward the scar zone after an MI [44,45], suggesting that the epicardium may play an important role in collateral artery formation.

Lymphangiogenesis has also been shown to play a critical role in cardiac repair [46]. After MI, mouse hearts showed the significant induction of lymphangiogenesis, which was stimulated by recombinant human vascular endothelial growth factor C (VEGF-C) treatment [46]. Furthermore, mouse hearts with stimulated lymphangiogenesis showed significantly improved cardiac function [46]. The induction of lymphangiogenesis after MI led to a reduction in immune cells seven days later, suggesting that newly formed lymph vessels aid in the resolution of inflammation after MI $\left[47^{\bullet *}\right]$. The genetic deletion of lymphatic vessel endothelial hyaluronan receptor 1 ( $L Y V E-1)$, which aids in the clearing of leukocytes from the injured region, led to diminished immune cell clearance and a worsened prognosis [47*0]. The cardiac interstitium, which is composed of fibroblasts, endothelial cells, stromal cells, and other cell types, is involved in homeostasis, the injury response, and the regenerative response, but the exact composition of the interstitium and the interplay between these cell types are poorly understood [48]. By using single-cell sequencing of non-CMs from homeostatic and injured hearts, distinct populations of myofibroblasts, tissue-resident macrophages, infiltrating macrophages, endothelial cells, and other cell types [49] were identified in injured hearts, suggesting the importance of interstitial cells in the injury and regenerative responses of the heart after MI. 


\section{Conclusions and future directions}

Recent studies have shed light on heart regeneration and have proposed promising new therapeutic approaches for effectively treating HF. Cardiac regeneration and repair can be achieved in animal models by stimulating endogenous CM renewal through the manipulation of molecular signals such as AAV virus-mediated Hippo inhibition, the manipulation of extracellular matrix by using a cardiac patch, the autologous exosome delivery of regenerative miRs, and immune modulation. However, careful consideration must be given to the limitations of these preclinical studies. Given the complex nature of the heart, a deeper understanding is needed of the intricate signaling and developmental pathways involved, as well as the different cardiac cell types and their interactions. This knowledge will be vital for developing specific and safe cardiac therapies.

\section{Acknowledgement}

Nicole Stancel, PhD, ELS(D), of Scientific Publications at the Texas Heart Institute, provided editorial support.

Funding statement

Supported by the National Institutes of Health (HL 127717, HL 130804, and HL 118761 to J.F.M.; R56HL142704, K01 DE026561, and R03DE025873 to J.W.), Department of Defense (CDMRP) W81XWH-16-PRMRP-IIRA (J.F.M.), MacDonald Research Fund Award 16RDM001 (J.F.M.), and the Vivian L. Smith Foundation (J.F.M.). J.F.M. was supported by the LeDucq Foundation's Transatlantic Networks of Excellence in Cardiovascular Research (14CVD01: "Defining the Genomic Topology of Atrial Fibrillation”). J.W. was supported by American Heart Association Scientist Development Grant 14SDG1984000.

\section{References and recommended reading}

Papers of particular interest, published within the period of review, have been highlighted as:

•• of outstanding interest

1. Leach JP, Martin JF: Cardiomyocyte proliferation for therapeutic regeneration. Curr Cardiol Rep 2018, 20:63. [PubMed: 29904823]

2. Benjamin EJ, Blaha MJ, Chiuve SE, Cushman M, Das SR, Deo R, de Ferranti SD, Floyd J, Fornage M, Gillespie C et al.: Heart disease and stroke statistics-2017 update: a report from the American Heart Association. Circulation 2017, 135:e146-e603. [PubMed: 28122885]

3. Bergmann O, Zdunek S, Felker A, Salehpour M, Alkass K, Bernard S, Sjostrom SL, Szewczykowska M, Jackowska T, Remedios C et al.: Dynamics of cell generation and turnover in the human heart. Cell 2015, 161:1566-1575. [PubMed: 26073943]

4. Hashimoto H, Olson EN, Bassel-Duby R: Therapeutic approaches for cardiac regeneration and repair. Nat Rev Cardiol 2018, 15:1-16. [PubMed: 29188810]

5. Ye L, D'Agostino G, Loo S, Wang C, Su L, Tan S, Tee G, Pua C, Pena E, Belen R et al.: Early regenerative capacity in the porcine heart. Circulation 2018, 138 CIRCULATIONAHA.117.031542.

6. Zhu W, Zhang E, Zhao M, Chong Z, Fan C, Tang Y, Hunter JD, Borovjagin AV, Walcott GP, Chen JY et al. : Regenerative potential of neonatal porcine hearts. Circulation 2018, 138 CIRCULATIONAHA.118.034886.

7. Porrello ER, Mahmoud AI, Simpson E, Hill JA, Richardson JA, Olson EN, Sadek HA: Transient regenerative potential of the neonatal mouse heart. Science 2011, 331:1078-1080. [PubMed: 21350179]

8. Wang J, Liu S, Heallen T, Martin JF: The Hippo pathway in the heart: pivotal roles in development, disease, and regeneration. Nat Rev Cardiol 2018, 15:1-13. [PubMed: 29188810] 
9. Xiao Y, Hill MC, Zhang M, Martin TJ, Morikawa Y, Wang S, Moise AR, Wythe JD, Martin JF: Hippo signaling plays an essential role in cell state transitions during cardiac fibroblast development. Dev Cell 2018, 45:153-169.e6. [PubMed: 29689192]

10. Leach JP, Heallen T, Zhang M, Rahmani M, Morikawa Y, Hill MC, Segura A, Willerson JT, Martin JF: Hippo pathway deficiency reverses systolic heart failure after infarction. Nature 2017, 550:260-264. [PubMed: 28976966] • This study shows, deletion of Salvador a Hippo pathway component in cardiomyocytes after an MI, leads to improvement in cardiac function.

11. Monroe TO, Hill MC, Morikawa Y, Leach JP, Heallen T, Cao S, Krijger P, de Laat W, Wehrens X, Rodney GG et al.: YAP partially reprograms chromatin accessibility to directly induce adult cardiogenesis in vivo. Dev Cell 2019, 48 765-779.e7. [PubMed: 30773489]

12. Stergachis AB, Neph S, Reynolds A, Humbert R, Miller B, Paige SL, Vernot B, Cheng JB, Thurman RE, Sandstrom R et al.: Developmental fate and cellular maturity encoded in human regulatory DNA landscapes. Cell 2013, 154:888-903. [PubMed: 23953118]

13. Morikawa Y, Heallen T, Leach J, Xiao Y, Martin JF: Dystrophin-glycoprotein complex sequesters Yap to inhibit cardiomyocyte proliferation. Nature 2017, 547:227-231. [PubMed: 28581498] •• This study demonstrates a direct interaction between Yap and DGC in restricting the cardiomyocyte proliferation.

14. Bassat E, Mutlak YE, Genzelinakh A, Shadrin IY, Umansky K, Yifa O, Kain D, Rajchman D, Leach J, Bassat D et al. : The extracellular matrix protein agrin promotes heart regeneration in mice. Nature 2017, 547:179-184. [PubMed: 28581497] •• The authors used an extracellular matrix protein Agrin, to trigger cardiomyocyte proliferation and cardiac regeneration through DGC-Yap interaction.

15. Ragni CV, Diguet N, Garrec J-F, Novotova M, Resende TP, Pop S, Charon N, Guillemot L, Kitasato L, Badouel C et al.: Amotl1 mediates sequestration of the Hippo effector Yap1 downstream of Fat4 to restrict heart growth. Nat Commun 2017, 8:14582. [PubMed: 28239148]

16. Tao G, Kahr PC, Morikawa Y, Zhang M, Rahmani M, Heallen TR, Li L, Sun Z, Olson EN, Amendt BA et al.: Pitx2 promotes heart repair by activating the antioxidant response after cardiac injury. Nature 2016, 534:119-123. [PubMed: 27251288]

17. von Gise A, Lin Z, Schlegelmilch K, Honor LB, Pan GM, Buck JN, Ma Q, Ishiwata T, Zhou B, Camargo FD et al. : YAP1, the nuclear target of Hippo signaling, stimulates heart growth through cardiomyocyte proliferation but not hypertrophy. Proc Natl Acad Sci USA 2012, 109:2394-2399. [PubMed: 22308401]

18. Li L, Tao G, Hill MC, Zhang M, Morikawa Y, Martin JF: Pitx2 maintains mitochondrial function during regeneration to prevent myocardial fat deposition. Development 2018, 145 pii: dev.168609.

19. Han Y, Chen A, Umansky K-BB, Oonk KA, Choi W-YY, Dickson AL, Ou J, Cigliola V, Yifa O, Cao J et al.: Vitamin D stimulates cardiomyocyte proliferation and controls organ size and regeneration in zebrafish. Dev Cell 2019, 48 853-863.e5. [PubMed: 30713073] •• This study used Zebrafish for screening small molecules and identified Vitamin-D as a potent inducer of cardiomyocyte proliferation.

20. Hirose K, Payumo AY, Cutie S, Hoang A, Zhang H, Guyot R, Lunn D, Bigley RB, Yu H, Wang J et al.: Evidence for hormonal control of heart regenerative capacity during endothermy acquisition. Science 2019, 364 eaar2038. [PubMed: 31624212] •• The authors revealed the activation thyroid hormone signaling axis as one of the factors responsible for cell cycle arrest and loss of regenerative potential in cardiomyocyte.

21. Patterson M, Barske L, Handel B, Rau CD, Gan P, Sharma A, Parikh S, Denholtz M, Huang Y, Yamaguchi $\mathrm{Y}$ et al. : Frequency of mononuclear diploid cardiomyocytes underlies natural variation in heart regeneration. Nat Genet 2017, 49 10.1038/ng.3929.

22. González-Rosa J, Sharpe M, Field D, Soonpaa MH, Field LJ, Burns CE, Burns GC: Myocardial polyploidization creates a barrier to heart regeneration in zebrafish. Dev Cell 2018, 44:433-446.e7. [PubMed: 29486195]

23. Puente BN, Kimura W, Muralidhar SA, Moon J, Amatruda JF, Phelps KL, Grinsfelder D, Rothermel BA, Chen R, Garcia JA et al.: The oxygen-rich postnatal environment induces cardiomyocyte cell-cycle arrest through DNA damage response. Cell 2014, 157:565-579. [PubMed: 24766806] 
24. Nakada Y, Canseco DC, Thet S, Abdisalaam S, Asaithamby A, Santos CX, Shah A, Zhang H, Faber JE, Kinter MT et al.: Hypoxia induces heart regeneration in adult mice. Nature 2016, 541:222-227. [PubMed: 27798600]

25. Mohamed T, Ang Y-S, Radzinsky E, Zhou P, Huang Y, Elfenbein A, Foley A, Magnitsky S, vastava D: Regulation of cell cycle to stimulate adult cardiomyocyte proliferation and cardiac regeneration. Cell 2018, 173.• This study used viral mediated over expression of cell cycle regulators to induce cardiomyocyte regeneration. And, used MADAM reporter mice to track cell division.

26. Huang W, Feng Y, Liang J, Yu H, Wang C, Wang B, Wang M, Jiang L, Meng W, Cai W et al.: Loss of microRNA-128 promotes cardiomyocyte proliferation and heart regeneration. Nat Commun 2018, 9:700. [PubMed: 29453456]

27. Borden A, Kurian J, Nickoloff E, Yang Y, Troupes CD, Ibetti J, Lucchese AM, Gao E, Mohsin S, Koch Wj et al. : Transient introduction of miR-294 in the heart promotes cardiomyocyte cell cycle reentry after injury. Circ Res 2019, 125:14-25. [PubMed: 30964391]

28. Lesizza P, Prosdocimo G, Martinelli V, Sinagra G, Zacchigna S, Giacca M: Single-dose intracardiac injection of proregenerative MicroRNAs improves cardiac function after myocardial infarction. Circ Res 2017, 120:1298-1304. [PubMed: 28077443]

29. Wang LL, Liu Y, Chung JJ, Wang T, Gaffey AC, Lu M, Cavanaugh CA, Zhou S, Kanade R, Atluri $P$ et al.: Sustained miRNA delivery from an injectable hydrogel promotes cardiomyocyte proliferation and functional regeneration after ischaemic injury. Nat Biomed Eng 2017, 1:983-992. [PubMed: 29354322] • The authors utilizes a shear-thinning hydrogel for miRNA delivery to achieve cardiomyocyte proliferation.

30. Gao F, Kataoka M, Liu N, Liang T, Huang Z-P, Gu F, Ding J, Liu J, Zhang F, Ma Q et al.: Therapeutic role of miR-19a/19b in cardiac regeneration and protection from myocardial infarction. Nat Commun 2019, 10:1802. [PubMed: 30996254]

31. Liu B, Lee BW, Nakanishi K, Villasante A, Williamson R, Metz J, Kim J, Kanai M, Bi L, Brown K et al.: Cardiac recovery via extended cell-free delivery of extracellular vesicles secreted by cardiomyocytes derived from induced pluripotent stem cells. Nat Biomed Eng 2018, 2:293-303. [PubMed: 30271672] •• This study delivered extracellular vesicles from iPS derived cardiomyocytes in a hydrogel patch to achieve heart regeneration.

32. Lai S-L, Marín-Juez R, Stainier DY: Immune responses in cardiac repair and regeneration: a comparative point of view. Cell Mol Life Sci 2019, 76:1365-1380. [PubMed: 30578442]

33. Hui SP, Sheng DZ, Sugimoto K, Gonzalez-Rajal A, Nakagawa S, Hesselson D, Kikuchi K: Zebrafish regulatory T cells mediate organ-specific regenerative programs. Dev Cell 2017, 43:659-672.e5. [PubMed: 29257949]

34. Zacchigna S, Martinelli V, Moimas S, Colliva A, Anzini M, Nordio A, Costa A, Rehman M, Vodret S, Pierro C et al.: Paracrine effect of regulatory T cells promotes cardiomyocyte proliferation during pregnancy and after myocardial infarction. Nat Commun 2018, 9:2432. [PubMed: 29946151] •• The authors revealed Treg cells are required for cardiomyocyte proliferation in neonatal stages and during pregnancy. And, injecting Tregs at the site of injury invokes a regenerative response.

35. Ramjee V, Li D, Manderfield LJ, Liu F, Engleka KA, Aghajanian H, Rodell CB, Lu W, Ho V, Wang $\mathrm{T}$ et al. : Epicardial YAP/TAZ orchestrate an immunosuppressive response following myocardial infarction. J Clin Invest 2017, 127:899-911. [PubMed: 28165342]

36. Hulsmans M, Clauss S, Xiao L, Aguirre AD, King KR, Hanley A, Hucker WJ, Wülfers EM, Seemann G, Courties G et al.: Macrophages facilitate electrical conduction in the heart. Cell 2017, 169:510-522.e20. [PubMed: 28431249]

37. Lai S-L, Marín-Juez R, Moura P, Kuenne C, Lai J, Tsedeke A, Guenther S, Looso M, Stainier DY: Reciprocal analyses in zebrafish and medaka reveal that harnessing the immune response promotes cardiac regeneration. eLife 2017, 6:e25605. [PubMed: 28632131]

38. Dick SA, Macklin JA, Nejat S, Momen A, Clemente-Casares X, Althagafi MG, Chen J, Kantores C, Hosseinzadeh S, Aronoff L et al.: Self-renewing residentcardiac macrophages limit adverse remodeling following myocardial infarction. Nat Immunol 2019, 20:29-39. [PubMed: 30538339] - This study used single cell sequencing to look at the heterogeneity of immune response at homeostasis and after an MI. 
39. Rutkowski MJ, Sughrue ME, Kane AJ, Ahn BJ, Fang S, Parsa AT: The complement cascade as a mediator of tissue growth and regeneration. Inflamm Res 2010, 59:897-905. [PubMed: 20517706]

40. Natarajan N, Abbas Y, Bryant DM, Gonzalez-Rosa J, Sharpe M, Uygur A, Cocco-Delgado LH, Ho N, Gerard NP, Gerard CJ et al. : Complement receptor C5aR1 plays an evolutionarily conserved role in successful cardiac regeneration. Circulation 2018, 137:2152-2165. [PubMed: 29348261]

41. Meoli DF, Sadeghi MM, Krassilnikova S, Bourke BN, Giordano FJ, Dione DP, Su H, Edwards SD, Liu S, Harris TD et al. : Noninvasive imaging of myocardial angiogenesis following experimental myocardial infarction. J Clin Invest 2004, 113:1684-1691. [PubMed: 15199403]

42. Marín-Juez R, Marass M, Gauvrit S, Rossi A, Lai S-L, Materna SC, Black BL, Stainier DY: Fast revascularization of the injured area is essential to support zebrafish heart regeneration. Proc Natl Acad Sci USA 2016, 113:11237-11242. [PubMed: 27647901]

43. Das S, Goldstone AB, Wang H, Farry J, D’Amato G, Paulsen MJ, Eskandari A, Hironaka CE, Phansalkar R, Sharma B et al.: A unique collateral artery development program promotes neonatal heart regeneration. Cell 2019, 176 1128-1142.e18. [PubMed: 30686582] •• This article showed that, the collateral artery formation after an MI promotes heart regeneration and mechanistically it is mediated by CXCL12 and CXCR4 signaling axis.

44. Cavallero S, Shen H, Yi C, Lien C-L, Kumar RS, Sucov HM: CXCL12 signaling is essential for maturation of the ventricular coronary endothelial plexus and establishment of functional coronary circulation. Dev Cell 2015, 33:469-477. [PubMed: 26017771]

45. Dubé KN, Thomas TM, Munshaw S, Rohling M, Riley PR, Smart N: Recapitulation of developmental mechanisms to revascularize the ischemic heart. JCI Insight 2017, 2:e96800.

46. Klotz L, Norman S, Vieira J, Masters M, Rohling M, Dubé KN, Bollini S, Matsuzaki F, Carr Ca, Riley PR: Cardiac lymphatics are heterogeneous in origin and respond to injury. Nature 2015, 522:62-67. [PubMed: 25992544]

47. Vieira J, Norman S, del Campo C, Cahill TJ, Barnette DN, Gunadasa-Rohling M, Johnson LA, Greaves DR, Carr CA, Jackson DG et al.: The cardiac lymphatic system stimulates resolution of inflammation following myocardial infarction. J Clin Invest 2018, 128:3402-3412. [PubMed: 29985167] • The authors revealed the importance of lymphatics in resolution of the initial immune response in the heart after an MI.

48. Forte E, Furtado M, Rosenthal N: The interstitium in cardiac repair: role of the immune-stromal cell interplay. Nat Rev Cardiol 2018, 15:1. [PubMed: 29188810]

49. Farbehi N, Patrick R, Dorison A, Xaymardan M, Janbandhu V, Wystub-Lis K, Ho JW, Nordon RE, Harvey RP: Single-cell expression profiling reveals dynamic flux of cardiac stromal, vascular and immune cells in health and injury. eLife 2019, 8:e43882. [PubMed: 30912746] 

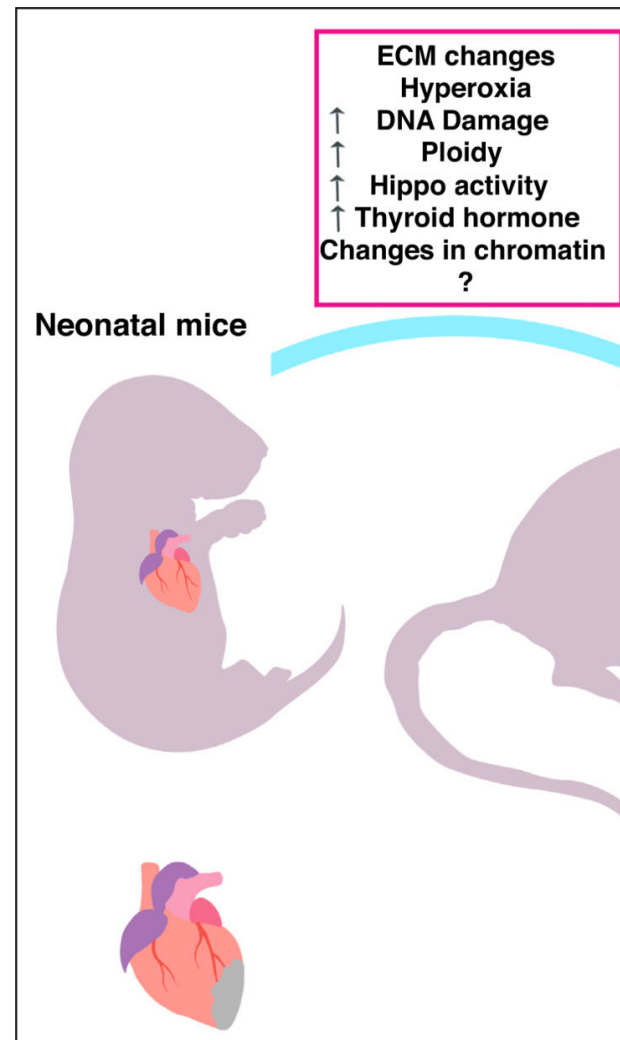

Cardiac injury

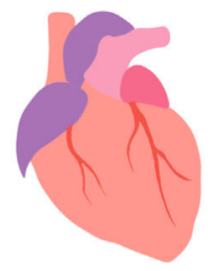

Cardiac regeneration
Adult mice

Figure 1.

Cardiac regeneration and injury model. Neonatal mice heart possesses remarkable regenerative capabilities. After an MI neonatal heart shows resolution of the scar and repopulation of the area with new cardiomyocytes. Adult mice heart loses the regenerative capabilities. After an injury adult heart shows formation of fibrotic scar. 


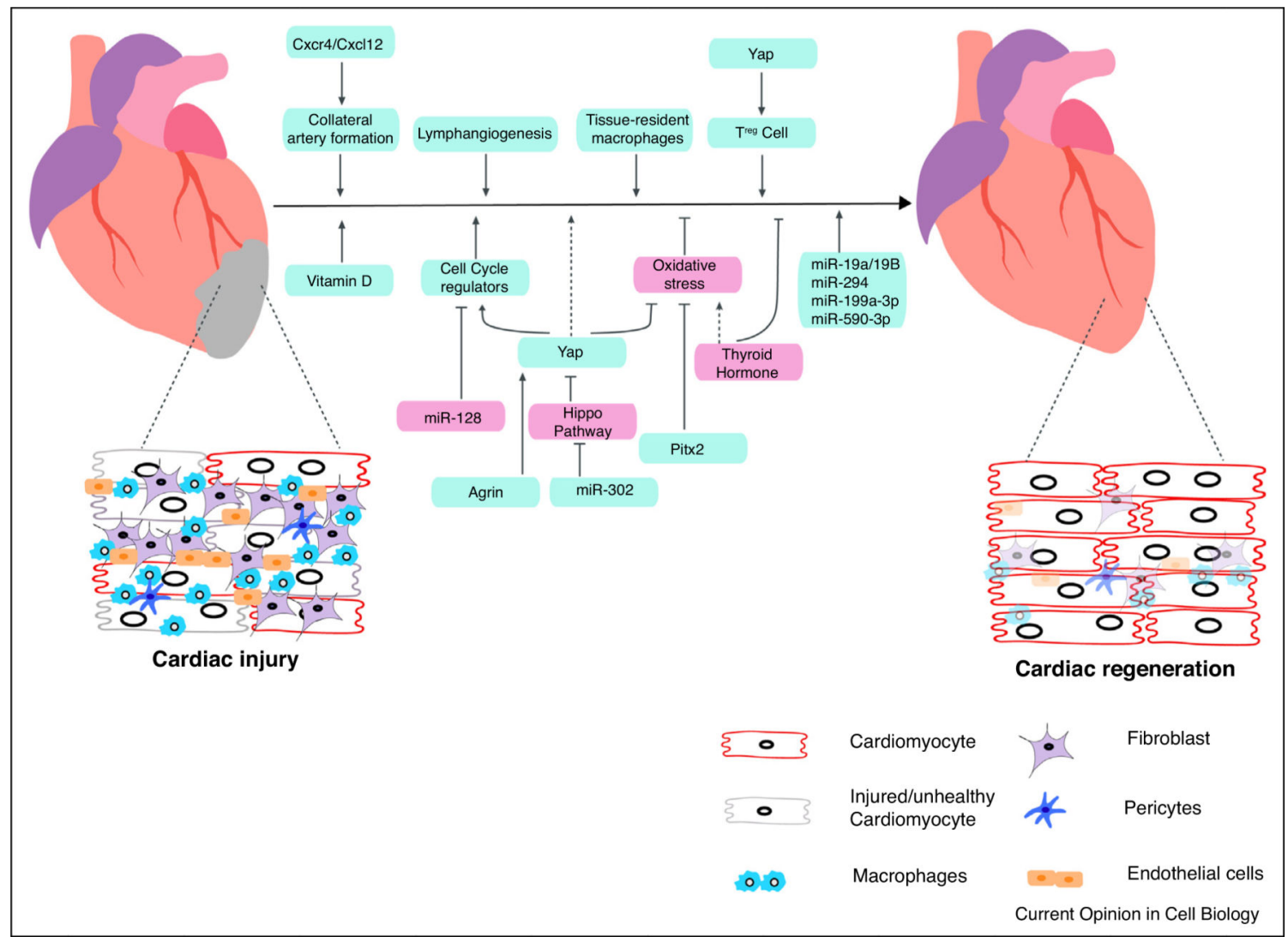

Figure 2.

Summary of recent advancement toward heart regeneration. A schematic representation of factors promoting (blue box) and inhibiting (pink box) cardiac regeneration. After an injury, scar area consists of unhealthy or dead cardiomyocytes, infiltrated with immune cells and repopulation of the scar are with fibroblast. Cardiomyocyte proliferation can be achieved through inhibition of Hippo pathway, expressing cell cycle regulators in cardiomyocytes, inhibition of thyroid hormone, manipulating extracellular matrix. Cardiac regeneration can also be achieved by activating heart resident macrophages, activation of $\mathrm{T}^{\mathrm{reg}}$ cell, increase in collateral artery and increased lymphogenesis. 